\title{
Identification of a Chemoresistant "Oxidative State-Low" Leukemic Subpopulation in CD34+ Human Acute Myeloid Leukemia
}

loannis Kotsianidis ${ }^{1 *}$, Dimitra Kokkinou ${ }^{2}$, Elena K Siapati ${ }^{3}$, Paraskevi Miltiades ${ }^{1}$, Eleftheria Lamprianidou' ${ }^{1}$, George Vassilopoulos ${ }^{3,4}$, Nicholas C Zoumpos ${ }^{1}$ and Alexandros Spyridonidis ${ }^{1}$

${ }^{1}$ Department of Hematology, Democritus University of Thrace, Medical school, Greece

${ }^{2}$ Division of Hematology, University Hospital of Patras, Greece

${ }^{3}$ Division of Genetics and Gene Therapy, Academy of Athens, Greece

${ }^{4}$ Division of Hematology, University Hospital of Thessaly, Greece

\begin{abstract}
Objective: Both normal and malignant stem cells maintain lower levels of reactive oxygen species (ROS), but the redox state in acute myeloid leukemia (AML) has not been thoroughly characterized and the role of ROS in leukemogenesis is still unclear. Herein, we report the identification of a rare but distinct ROS low subpopulation in primary CD34+ AML samples.
\end{abstract}

Methods: We analysed the ROS state of a number of AML samples by flow cytometry using the redox-sensitive fluorescence dye 2'7;-dichlorodihydrofluorescein diacetate. We FACS-sorted the ROS ${ }^{\text {low }}$ cells and investigated their immunophenotype, in vivo engraftment potential as well as their ability to withstand chemotherapeutic treatment.

Results: Compared to the total CD34+ cells the ROS low subset contained significantly more CMP-like and less GMP-like progenitors and could establish leukemia in NOD/SCID mice. Additionally, ROS ${ }^{\text {low }}$ cells bore a chemoresistant phenotype as they were more quiescent than total CD34+ AML cells, and showed increased in vitro chemoresistance and markedly higher GM-CSF-induced phosphorylation of STAT5.

Conclusions: Thus, the ROS ${ }^{\text {low }}$ subpopulation arises as a novel candidate for cell-specific therapeutic targeting in AML. Further studies will help to ascertain the exact role of the ROS ${ }^{\text {low }}$ subset in the pathobiology and clinical management of AML.

Keywords: AML; Oxidative stress; Leukemia stem cells; ROS; NOD/ SCID; Chemoresistance; CMP; GMP

\section{Introduction}

Reactive oxygen species (ROS) are oxygen-containing side products of normal aerobic metabolism. Although it was initially believed that ROS constitute only damaging by-products, recent evidence suggest that ROS actively participate in signal transduction pathways and regulate cell survival and proliferation in a dose-depended manner $[1,2]$. Endogenous intracellular redox state is tightly controlled by free radical scavenging systems and the appropriate ROS levels differ among cell types and differentiation status [3]. Hematopoietic stem cells (HSCs) in mice reside mainly in the $\mathrm{ROS}^{\text {low }}$ subset of hematopoietic progenitors [4], whereas forced increase of ROS in human Lin CD $34^{+} \mathrm{CD} 38^{-}$cord blood cells leads to loss of stem cell activity [5-7]. Similarly, though increased ROS levels in tumor cells have been implicated in cancer initiation and progression.

Cancer stem cells (CSCs) maintain a low redox status, apparently through upregulation of anti-oxidative enzymes [1,2,8]. Elevated ROS levels drive out of quiescence both normal and clonal stem cells, while administration of $\mathrm{N}$-acetylcysteine, an antioxidant, can partially restore the self-renewal capacity [9]. Moreover, ROS production via constitutive activation of the Ras oncogene appears to drive the leukemic progression [10]. In acute myeloid leukemia (AML) free radicals, antioxidant enzymes and oxidative damage products are elevated, but the redox state in leukemia blasts has not been thoroughly characterized and the role of ROS in leukemogenesis is still unclear [11-14]. Most adults with acute myeloid leukemia (AML) are not cured due to chemoresistance or relapse. AML may be organized in a similar way to normal hematopoiesis and, hence, disease recurrence could be due to a subset of leukemic stem cells (LSC) [15]. Here, we assessed ROS levels in primary AML blasts and we identified a distinct
ROS $^{\text {low }}$ subpopulation with a quiescent, chemoresistant phenotype and leukemia-initiating cell activity (LIC).

\section{Methods}

\section{Patients}

Bone marrow (BM) or peripheral blood (PB) was obtained from 44 newly diagnosed, $\mathrm{CD} 34^{+} \mathrm{AML}$ patients (Table 1) after informed consent and Institutional Review Board approval. AML cell lines (U937, THP1) were obtained from DSMZ (Braunschweig, Germany). Mononuclear cells were isolated over Ficoll-Hypaque and used either immediately or after cryopreservation.

\section{Cell isolation and assessment of ROS levels and cell cycle status}

FACS-sorting of total CD $34^{+}$and $\mathrm{ROS}^{\text {low }}$ live cells was performed by using a FACSVantage SE cytometer (Becton Dickinson). Assessment of ROS levels in sorted subpopulations was done using the redox-

*Corresponding author: loannis Kotsianidis, MD, PhD, Department of Hematology, Democritus University of Thrace, Medical School, Dragana, Alexandroupolis 68100, Greece, Tel: +302551030320; Fax: +302551076154 E-mail: ikotsian@med.duth.gr, jankots@yahoo.gr

Received August 04, 2014; Accepted September 23, 2014; Published September 25, 2014

Citation: Kotsianidis I, Kokkinou D, Siapati EK, Miltiades P, Lamprianidou E, et al. (2014) Identification of a Chemoresistant "Oxidative State-Low" Leukemic Subpopulation in CD34+ Human Acute Myeloid Leukemia. J Stem Cell Res Ther 4: 235. doi:10.4172/2157-7633.1000235

Copyright: (c) 2014 Kotsianidis I, et al. This is an open-access article distributed under the terms of the Creative Commons Attribution License, which permits unrestricted use, distribution, and reproduction in any medium, provided the original author and source are credited. 
Page 2 of 9

\begin{tabular}{|c|c|c|c|c|c|c|c|c|c|c|}
\hline $\begin{array}{l}\mathrm{Pt} \\
\text { No }\end{array}$ & Age & Sex & Diagnosis & Source & Cytogenetics & $\begin{array}{l}\text { FLT3/ITD } \\
\text { mutation }\end{array}$ & Induction chemo & $\begin{array}{c}\text { Response } \\
Y=C R \\
N=\text { No CR }\end{array}$ & $\begin{array}{l}\text { ROS low } \\
\% \text { of total } \\
\text { CD34+ }\end{array}$ & $\begin{array}{c}\text { ROS Relative } \\
\text { MFI }\end{array}$ \\
\hline 1 & 60 & $\mathrm{~F}$ & SAML & PB & $\operatorname{del}(5 q)$ & negative & $7+3$ regimen & $\mathrm{N}$ & 2.08 & 0.604 \\
\hline 2 & 82 & $\mathrm{~F}$ & SAML & PB & $N / D$ & N/D & No treatment & N/A & 0.24 & 8.853 \\
\hline 3 & 65 & $\mathrm{~F}$ & de novo AML & BM & normal & negative & $7+3$ regimen & $\mathrm{Y}$ & 0.58 & 2.259 \\
\hline 4 & 56 & $M$ & SAML & BM & complex & negative & $7+3$ regimen & $\mathrm{N}$ & 0.69 & 3.777 \\
\hline 5 & 68 & $M$ & SAML & PB & trisomy 8 & $N / D$ & 5-azacitidine & $\mathrm{N}$ & 2.18 & 2.639 \\
\hline 6 & 69 & M & SAML & PB & normal & $N / D$ & 5-azacitidine & $\mathrm{N}$ & 3.11 & 1.283 \\
\hline 7 & 50 & $M$ & de novo AML & PB & $\operatorname{inv}(16)(p 13.1 q 22)$ & negative & $7+3$ regimen & $Y$ & 0.34 & 3.741 \\
\hline 8 & 74 & $M$ & de novo AML & BM & $N / D$ & $N / D$ & No treatment & $\mathrm{N}$ & 0.3 & 3.051 \\
\hline 9 & 66 & $\mathrm{~F}$ & de novo AML & PB & $\operatorname{del}(20 q)$ & $N / D$ & $7+3$ regimen & $\mathrm{N}$ & 2.34 & 11.095 \\
\hline 10 & 74 & $\mathrm{~F}$ & de novo AML & PB & N/A & positive & $7+3$ regimen & N & 0.31 & 6.001 \\
\hline 11 & 77 & $M$ & de novo AML & PB & N/D & positive & LD-AraC & $\mathrm{N}$ & 0.6 & 1.520 \\
\hline 12 & 72 & $M$ & de novo AML & PB & normal & positive & $7+3$ regimen & $Y$ & 0.4 & 3.839 \\
\hline 13 & 73 & $M$ & SAML & PB & trisomy 8 & negative & $7+3$ regimen & $Y$ & 1.41 & 1.270 \\
\hline 14 & 78 & $M$ & de novo AML & PB & $N / D$ & positive & $7+3$ regimen & N & 1.92 & 1.294 \\
\hline 15 & 39 & $M$ & de novo AML & PB & normal & $N / D$ & $7+3$ regimen & $Y$ & 0.29 & 2.460 \\
\hline 16 & 76 & $\mathrm{~F}$ & de novo AML & PB & N/A & $N / A$ & $N / A$ & N/A & 1.27 & 3.102 \\
\hline 17 & 51 & $\mathrm{~F}$ & de novo AML & PB & monosomy 7 & negative & $7+3$ regimen & $\mathrm{N}$ & 0.84 & 2.360 \\
\hline 18 & 35 & $M$ & de novo AML & BM & normal & $N / D$ & $7+3$ regimen & $Y$ & 2.69 & 8.991 \\
\hline 19 & 25 & $\mathrm{~F}$ & de novo AML & PB & $\operatorname{inv}(16)(p 13.1 q 22)$ & negative & $7+3$ regimen & $Y$ & 0.41 & 3.236 \\
\hline 20 & 77 & $\mathrm{M}$ & de novo AML & BM & $\mathrm{N} / \mathrm{A}$ & negative & $\mathrm{N} / \mathrm{A}$ & $\mathrm{N} / \mathrm{A}$ & 2.17 & 3.819 \\
\hline 21 & 66 & $M$ & de novo AML & PB & normal & negative & $7+3$ regimen & $\mathrm{N}$ & 0.61 & 8.093 \\
\hline 22 & 76 & $\mathrm{~F}$ & de novo AML & PB & N/A & positive & No treatment & N/A & 0.35 & 2.698 \\
\hline 23 & 75 & $\mathrm{~F}$ & sAML & BM & N/D & $\mathrm{N} / \mathrm{D}$ & No treatment & $\mathrm{N} / \mathrm{A}$ & 4.99 & 5.720 \\
\hline 24 & 81 & $\mathrm{~F}$ & de novo AML & PB & $\mathrm{N} / \mathrm{A}$ & positive & LD-AraC & $\mathrm{N}$ & 0.2 & 1.722 \\
\hline 25 & 56 & M & de novo AML & PB & $N / A$ & $N / D$ & $7+3$ regimen & $\mathrm{N}$ & 0.4 & 11.529 \\
\hline 26 & 77 & $\mathrm{M}$ & de novo AML & PB & $\mathrm{N} / \mathrm{A}$ & $\mathrm{N} / \mathrm{A}$ & No treatment & $\mathrm{N} / \mathrm{A}$ & 0.34 & 3.340 \\
\hline 27 & 75 & $M$ & sAML & PB & $\mathrm{N} / \mathrm{A}$ & $\mathrm{N} / \mathrm{A}$ & No treatment & $\mathrm{N} / \mathrm{A}$ & 1.2 & 6.484 \\
\hline 28 & 49 & M & SAML & PB & monosomy 7 & $N / D$ & $7+3$ regimen & $\mathrm{N}$ & 0.52 & 3.965 \\
\hline 29 & 83 & $\mathrm{M}$ & sAML & PB & normal & N/D & LD-AraC & $\mathrm{N}$ & 0.79 & 6.090 \\
\hline 30 & 60 & $M$ & de novo AML & BM & $N / D$ & N/D & No treatment & $\mathrm{N} / \mathrm{A}$ & 0.52 & 7.919 \\
\hline 31 & 76 & $\mathrm{~F}$ & de novo AML & PB & $N / D$ & N/D & No treatment & N/A & 0.8 & 1.389 \\
\hline 32 & 68 & $\mathrm{M}$ & de novo AML & PB & complex & $N / D$ & No treatment & $\mathrm{N} / \mathrm{A}$ & 0.5 & 1.811 \\
\hline 33 & 63 & $\mathrm{M}$ & de novo AML & BM & normal & negative & $7+3$ regimen & N & 0.2 & 3.969 \\
\hline 34 & 78 & M & SAML & PB & $N / A$ & $N / A$ & No treatment & $N / A$ & 1.22 & 6.427 \\
\hline 35 & 72 & $\mathrm{~F}$ & de novo AML & BM & normal & $\mathrm{N} / \mathrm{A}$ & No treatment & N/A & 1.4 & 2.366 \\
\hline 36 & 68 & M & sAML & PB & del(12)(p11.2) & N/D & 5-azacitidine & $\mathrm{N}$ & 1.5 & 2.899 \\
\hline 37 & 74 & M & SAML & BM & normal & $N / D$ & 5-azacitidine & $\mathrm{N}$ & 0.43 & 0.789 \\
\hline 38 & 67 & $M$ & de novo AML & BM & normal & $\mathrm{N} / \mathrm{A}$ & $7+3$ regimen & $\mathrm{N}$ & 0.7 & 3.415 \\
\hline 39 & 66 & M & de novo AML & PB & $\begin{array}{l}\text { Monosomy } 7 \mathrm{q} \\
\text { trisomy 1q }\end{array}$ & $\mathrm{N} / \mathrm{A}$ & $7+3$ regimen & $\mathrm{N}$ & 1 & 6.335 \\
\hline 40 & 69 & $M$ & sAML & PB & normal & $N / D$ & 5-azacitidine & $\mathrm{N}$ & 0.5 & 5.292 \\
\hline 41 & 51 & $\mathrm{~F}$ & de novo AML & PB & $\operatorname{inv}(16)(p 13.1 q 22)$ & negative & $7+3$ regimen & $Y$ & 0.78 & 4.43 \\
\hline 42 & 72 & M & SAML & PB & N/A & $N / D$ & 5-azacitidine & $\mathrm{N}$ & 0.81 & 6.28 \\
\hline 43 & 60 & $M$ & de novo AML & BM & normal & negative & $7+3$ regimen & $\mathrm{N}$ & 0.2 & 4.62 \\
\hline 44 & 48 & M & de novo AML & BM & normal & negative & $7+3$ regimen & $\mathrm{N}$ & 0.4 & 5.02 \\
\hline
\end{tabular}

Table 1: Parameters of patients included in study. AML, acute myeloid leukemia; CG, cytogenetics; F, female; M, male; MDS, myelodysplastic syndrome; sAML, AML evolving from antecedent MDS; N, normal; N/A, not available; N/D, not done.

sensitive fluorescence dye 2'7;-dichlorodihydrofluorescein diacetate (DCF, Molecular Probes Inc) as previously described [16]. Relative ROS levels in AML blasts were determined as the ratio of the Mean Fluorescence Intensity (MFI) values of DCF in AML CD34+ cells to the MFI of total lymphocytes. For cell-cycle analysis sorted subsets were stained with Ki-67 and 7-AAD after fixation and permeabilization with Cytofix/CytoPerm (BD Biosciences).

\section{Antibodies, data acquisition and analysis}

The following antibodies and fluorescent compounds were used: CD2 (clone RPA-2.10), CD3 (HIT3a), CD4 (RPA-T4), CD8 ( RPA-T8), CD19 (HIB19), CD20 (2H7), GPA (GA-R2), CD90 (5E10), CD34 (8G12), CD56 (NCAM 16.2) and CD45 (2D1), Ki-67 (B56), annexin $\mathrm{V}$, propidium iodide (PI) and 7-aminoactinomycin D dye (7AAD), all from BD Biosciences; CD45 (J33) and CD38 (LS198.4.3) from Beckman Coulter; CD123 (6H6), and CD45RA (HI100) from Biolegend). Data 
acquisition was performed on a 5-color EPICS XL (Coulter, Fullerton, CA, USA) and a 4-color FACSCalibur (BD Biosciences, CA, USA) cytometers and analyses were done by using Flowjo software (Treestar, Ashland, OR).

\section{Fluorescence in situ hybridization (FISH)}

FISH was performed in sorted ROS ${ }^{\text {low }}$ live cells according to standard protocols, using the Vysis D7S522/CEP7 FISH Probe Kit. In total, approximately 200 nuclei were scored in each of 3 patients bearing the -7 cytogenetic abnormality.

\section{Immunophenotypic analysis of leukemic hematopoietic hierarchy}

Cells expressing mature lineage markers were depleted from mononuclear cells after staining with an antibody cocktail consisting of anti-CD2, CD3, CD4, CD8, CD19, CD20 and GPA [17] and antiPE immunomagnetic MicroBeads (Miltenyi Biotec). Purified Lin cells were then subjected to 5-color immunophenotypic analysis to define the maturation stage of $\mathrm{CD} 34^{+} \mathrm{AML}$ and ROS ${ }^{\text {low }}$ cells by concomitant staining with DCF and CD34, CD38, CD90, CD123 and CD45RA.

\section{In vitro chemoresistance assay in total CD34+ blasts}

Briefly, $10^{6} / \mathrm{ml}$ total AML cells were cultured in triplicates for $24 \mathrm{~h}$ in standard medium (RPMI 1640 \& 10\% FBS) with or without increasing concentrations of daunorubicin (DNR, 0.5-2 $\mu \mathrm{M}$, Pharmacia \& Upjohn, Milan, Italy) or cytarabine (AraC, 2.5-10 $\mu \mathrm{M}$, Pfizer, Milan, Italy) as previously described [18]. Drug-induced death and apoptosis were assessed by flow cytometry using Annexin-V/Propidium Iodide staining.

\section{Xenograft assay}

The in-vivo engraftment capacity of sorted cells was analyzed as described elsewhere [19]. Briefly, cells $\left(2 \times 10^{5}\right)$ ROS low cells or $10^{6}-10^{7}$ bulk cells from each patient were intravenously injected into non-obese diabetic severe combined immunodeficient (NOD-SCID) mice and leukemic engraftment was assessed 12 weeks after transplantation.

\section{Single-cell phosphospecific flow cytometry}

For the determination of the levels of phosphorylated pSTAT-3 and pSTAT-5, sorted total CD $34^{+}$AML and ROS low cells were either left untreated, or were stimulated with Granulocyte-Colony Stimulating Factor (G-CSF) or Granulocyte Macrophage Stimulating Factor (GM$\mathrm{CSF}$ ) (Miltenyi Biotec $\mathrm{GmbH}$, Germany) for 15 minutes at $37^{\circ} \mathrm{C}$. Stimulation was halted by fixation with Cytofix Fixation Buffer (BD Biosciences) and then cells were permeabilized with Perm Buffer III (BD Biosciences) and stained with the following antibodies: CD34 (clone 8G12), phospho-STAT3 (clone Y705), and phospho-STAT5 (clone Y694). Fluorescence minus one (FMO) controls were used and basal phosphorylation levels of pSTAT3 and pSTAT5 were expressed as log2 [Median fluorescence intensity (MFI) (unstimulated)/MFI (FMO)] and potentiated levels as $\log 2$ [MFI (stimulated)/MFI (unstimulated)].

\section{Statistical analysis}

All analyses were performed using SPSS 17.0 software (SPSS Science, Chicago, IL). Data are presented as mean \pm SEM. The significance of the differences was assessed by unpaired or paired Student's $t$ test and one-way ANOVA as appropriate. Correlations of ROS MFI and the percentage of $\operatorname{ROS}^{\text {low }}$ subset with age $(n=44)$, karyotype $(n=26)$ and response to initial treatment $(n=30)$ were performed using Pearson's correlation.

\section{Results}

\section{ROS content in primary AML cells}

Intracellular ROS levels of FACS-sorted CD $34^{+} \mathrm{AML}$ cells exhibited a wide variation among the patient samples (median relative MFI: 3.41, range $0.6-11.52$, Figure 1a). In order to test the potential interference of exogenous factors in the fluorocytometric assessment of ROS using the DCF dye, e.g. the immediate exposure of cells to higher oxygen after isolation, or modulation by the activity of P-glycoprotein (P-gp) [20], we performed repeated measurements in a) paired samples before and after the 2-hour sorting procedure, $b$ ) in paired fresh and freeze-thawed samples and c) upon treatment with the P-glycoprotein efflux pump inhibitor cyclosporine A (supplementary Figure 1). All measurements showed little variation confirming that the heterogeneous DCF pattern in AML cells reliably reflects the endogenous redox status of AML blasts.

It has been reported that internal tandem duplication of the FLT3 gene (FLT3-ITD) increases ROS production potentially via overactive STAT5 signaling [11], thus the heterogeneity of ROS content may, at least in part, mirror the diversity of oncogenic signaling pathways. Nevertheless, we found no differences in ROS levels between FLT3-ITD positive and negative AML patients ( $\mathrm{p}=0.4$, Figure 2). Likewise, there was no association of ROS levels with age, karyotype, AML subtype, i.e. secondary or de novo, and response to induction chemotherapy (Figure 2a-c).

\section{Identification and characterization of a novel subpopulation of ROS ${ }^{\text {low }}$ AML blasts}

Unexpectedly, we noticed that in contrast to the AML cell lines U937 and THP-1, both of which expressed ROS homogeneously, primary blasts showed a diversified DCF/DA staining with a discrete fraction of ROS ${ }^{\text {low }}$ cells at the left tail of each distribution (median $=0.61 \%$ of $\mathrm{CD}^{+} 4^{+}$cells, range $0.2 \%$ to $4.99 \%$, Figure $1 \mathrm{~b}$ ). To confirm that the ROS ${ }^{\text {low }}$ subpopulation contained exclusively leukemic cells and did not represent contaminating normal cell populations or debris, we FACS-sorted ROS ${ }^{\text {low }}$ events from 3 individuals with AML and subjected them to morphological evaluation after staining with May-Grünwald Giemsa, cytometric analysis of aberrant surface marker expression and fluorescence in situ hybridization (FISH) analysis of specific cytogenetic abnormalities. All analyses confirmed the clonal nature of ROS ${ }^{\text {low }}$ cells (Figure 1c-f).

Similar to the intracellular ROS levels, the prevalence of ROS low cells did not differ based on age, gender, karyotype, FLT3-ITD status, and response to initial treatment (Figure 2a-d). By contrast, patients with secondary AML evolving from myelodysplastic syndrome (MDS) displayed a twofold increase of $\mathrm{ROS}^{\text {low }}$ subpopulation compared with de novo AML patients $(1.44 \% \pm 0.32 \%$ vs. $0.78 \pm 0.12 \%$, respectively, $p=0.029$, Figure $2 \mathrm{~d}$ ). Notably, the ROS levels and the percentage of ROS $^{\text {low }}$ subset were not intercorrelated and were both identical among BM and PB (supplementary Figure 2).

Recent findings challenge the leukemia stem cell (LSC) model and suggest that in approximately $90 \%$ of $\mathrm{CD} 34^{+}$AML cases LSCs reside in the granulocyte-macrophage progenitor (GMP) and lymphoidprimed multipotential progenitor (LMPP) compartments (LMPP/ GMP-like AML) [17]. We observed that in five LMPP/GMP-like AML samples, the ROS ${ }^{\text {low }}$ fraction contained significantly more CMP-like $(\mathrm{p}=0.04)$ and less GMP-like cells $(\mathrm{p}=0.03)$ compared to the bulk AML population. Also, LMPP-like cells were lower in the ROS low subset, 
a

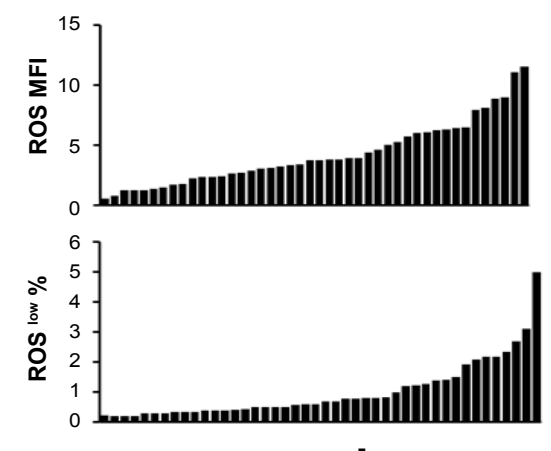

C

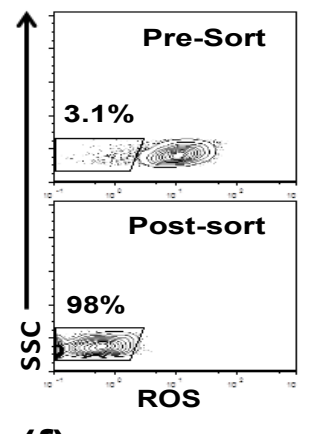

d

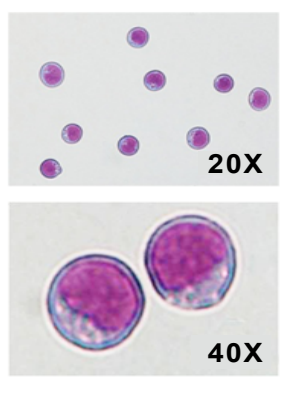

b

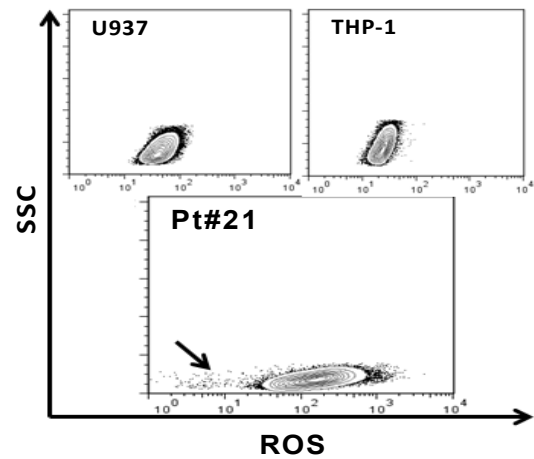

e

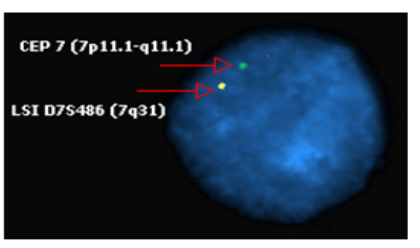

(f)

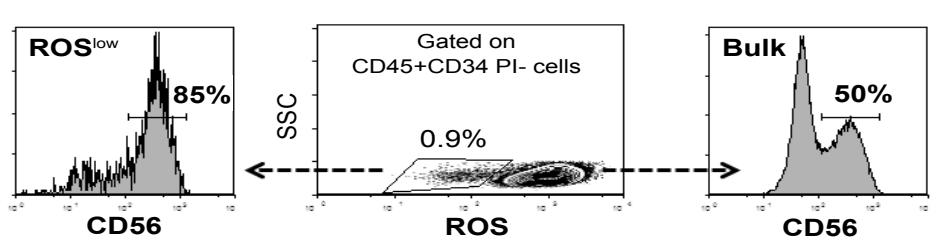

Figure 1: Interpatient variability of redox state in AML cells and identification of a novel "oxidative state-low" leukemic subpopulation. (a) Waterfall plots illustrating the distribution of values for ROS mean fluorescence intensity (MFI) and the prevalence of ROSlow cells expressed as percentage of total CD34+ live cells. (b) Representative flow cytometry plots of DCF/DA staining in U937 and THP-1 AML cell lines (performed in triplicates, upper plots) and in primary AML blasts (bottom plot, gated on CD45lowCD34+ live cells) showing a discrete ROSlow subpopulation only in the latter sample (arrow). (c) FACS sorting of the ROSlow subpopulation of AML blasts. Plots are gated on CD45lowCD34+ live cells. (d) Sorted ROSlowCD45lowCD34+ cells were morphologically consistent with blasts and (e) bore the characteristic cytogenetic abnormality (monosomy 7 ), as confirmed by FISH analysis which revealed single hybridization signal for each chromosome 7 probe (regions $7 q 31$ and $7 p 11.1-q 11.1$ ) in 93.6\% of nuclei. (f) The ROSlow subset is enriched in clonal cells as indicated by the high expression of the aberrant marker CD56 in 3 AML patients. Sorted ROSlow cells were stained with May-Grünwald-Giemsa. Photographs were taken by using a NIKON Eclipse TE2000U Inverted microscope; $20 x$ and 40x magnifications are shown.

while megakaryocyte-erythroid progenitor (MEP)-like cells were higher, but statistical significance was not reached for both progenitor types (Figure 3a-c). Of note, CD38 expression was identical among ROS $^{\text {low }}$ and total leukemia blasts (supplementary Figure 3). Although these results suggest that the ROS ${ }^{\text {low }}$ subset does not reside in the recently proposed LSC compartment of LMPP/GMP-like AML, when we assessed LIC activity in 16 AML samples by transplanting both sorted ROS low and total leukemia blasts from each patient in NOD/ SCID mice we observed identical levels of primary engraftment in 3/16 samples (median values: ROS ${ }^{\text {low }} 1.4 \%$ vs. total blasts $1.5 \%$ ), indicating that $\operatorname{ROS}^{\text {low }}$ cells have potentially comparable leukemogenic potential with the bulk leukemic population (Figure 4a).

Next, we evaluated quiescence and chemoresistance, two cardinal properties of leukemia propagating cells, in sorted $\mathrm{ROS}^{\text {low }}$ cells. Cell cycle analysis revealed that significantly more ROS ${ }^{\text {low }}$ cells resided in the G0 phase compared to the bulk leukemic population $(70 \% \pm 7.4 \%$ vs. $51 \% \pm 9.2 \%$, respectively, $\mathrm{p}=0.027$, Figure $4 \mathrm{~b}-\mathrm{c}$ ). Additionally, in in vitro chemosensitivity assays, sorted ROS $^{\text {low }}$ cells exhibited significantly increased chemoresistance in daunorubicin and cytarabine compared to the bulk AML population ( $\mathrm{p}=0.02$, Figure $4 \mathrm{~d}$ ), whereas in a different set of experiments performed by using total blasts only, the proportion of ROS ${ }^{\text {low }}$ viable, non-apoptotic cells increased significantly after culture with either daunorubicin or cytarabine (supplementary Figure 4).

G-CSF and GM-CSF-induced overexpression of the phosphorylated form of STAT5 in AML blasts associates with resistance to induction chemotherapy [21,22], whereas the magnitude of GM-CSF-induced activation of pSTAT5 in Juvenile Chronic Myeloid Leukemia, a typically chemoresistant malignancy, correlates with disease status [23]. Using single-cell phosphospecific flow cytometry we observed a marked GMCSF-induced upregulation of pSTAT5 in ROS ${ }^{\text {low }}$ cells compared to the bulk AML cells, consistent with a chemoresistant phenotype of ROS ${ }^{\text {low }}$ subset ( $\mathrm{p}=0,02$, Figure $4 \mathrm{e}$ and supplementary Figure 5). By contrast, no differences in basal and G-CSF-induced levels of both pSTAT5 and pSTAT3 were noticed among the two cell populations (Figure $4 \mathrm{e}$ and supplementary Figure 5). 
a

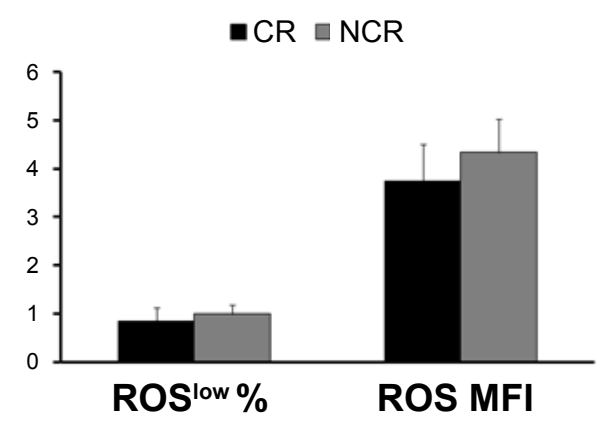

C

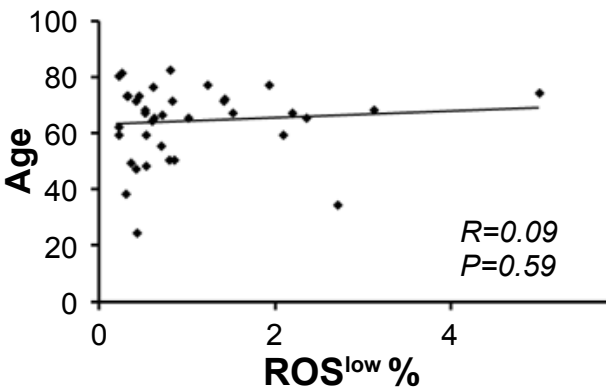

d

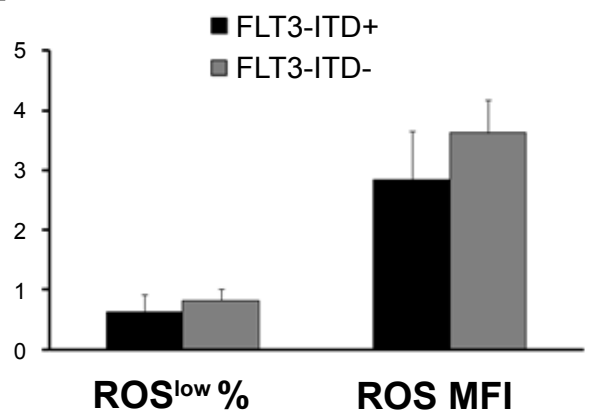

b

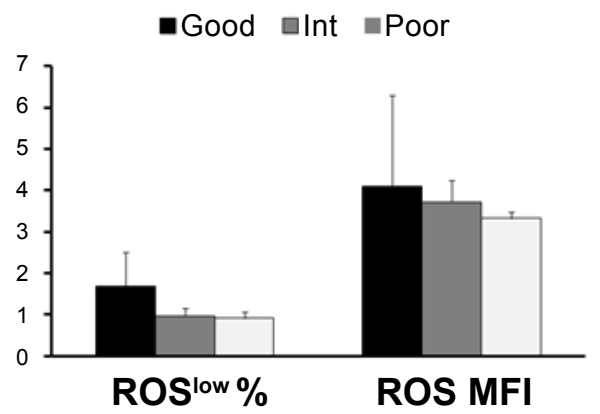

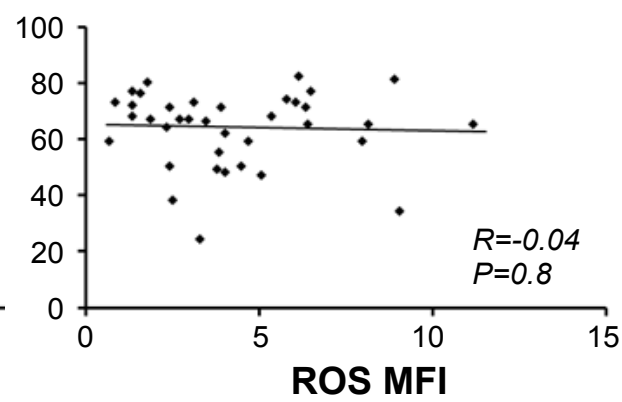

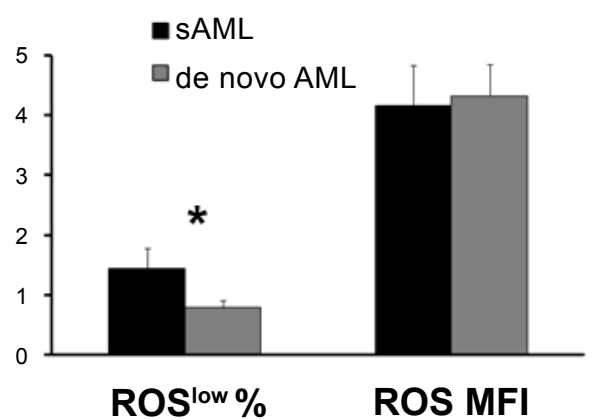

Figure 2: The percentage of ROSlow cells and the ROS levels in CD34+ blasts are not associated with age, karyotype and response to induction chemotherapy. (a) Both the relative ROS levels in AML blasts and the percentage of ROS low subpopulation were identical among patients with complete remission after induction therapy $(C R, n=9)$ and those who failed $C R(n=21, p=0.65$ and $p=0.6$, respectively). The classic " $7+3$ " regimen was administered as induction in all patients. (b) Likewise, no association exists between either ROS levels $(p=0.67)$ or the percentage of ROS ${ }^{\text {low }}$ cells $(p=0.43)$ and the cytogenetic risk (good $=4$, intermediate $=16$, poor $=6$ patients, respectively). (c) No correlation of both ROS MFI and the percentage of ROS low cells with the age of the patients ( $n=44)$. (d) Similar prevalence of ROS low cells and intracellular ROS levels in CD34+ blasts of FLT3-ITD positive $(n=6)$ and negative $(n=13)$ patients with de novo AML (left graph). By contrast, significantly higher levels of ROS low blasts in patients with secondary AML $(n=15)$ evolving from MDS compared to de novo AML blasts $(n=29)$, whereas the endogenous ROS content of CD34+ blasts was identical among the two disorders (right graph). Data are expressed as mean $\pm \mathrm{SEM}$; * $\mathrm{P}<0.05$ by unpaired t-test.

\section{Discussion}

The concept of leukemia stem cells which are able to initiate and propagate the disease is now well established [24]. Accurate identification and targeting of LSCs is of obvious significance for the effective treatment and the complete eradication of the leukemic clones. However, the characterization of LSCs by using surface markers is highly inconsistent among the various research groups, whereas novel data suggest that LSC may even share a progenitor rather than a stem cell phenotype $[17,25,26]$. Numerous data are now accumulating on the role of energy metabolism and in particular the redox state of cancer stem cells. Recently is has been shown that, similar to the normal HSCs in mice, most LSCs in human AML have low ROS levels [27]. However, no distinct subpopulation with low redox levels was defined. In the present work, we report for the first time the identification of a novel rare subpopulation of $\mathrm{CD} 34^{+} \mathrm{ROS}^{\text {low }} \mathrm{AML}$ cells with a chemoresistant, LSC-like phenotype.

In line with recent findings [27], we observed high interpatient variability of ROS content in AML CD $34^{+}$cells. The biology under the heterogeneity of ROS staining is obscure. Although leukemogenic pathways via Ras [10] or FLT3 mutations [28] have been associated with increased redox state, we were not able to find a correlation of ROS levels with either the FLT3/ITD mutation, or with clinical parameters of disease aggressiveness. Though the limited size of our cohort weakens the statistical power of the above correlations, ROS 
a

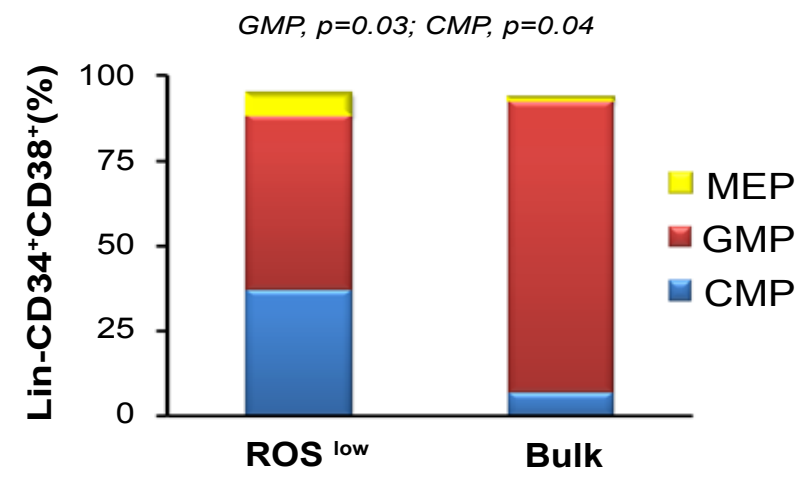

b

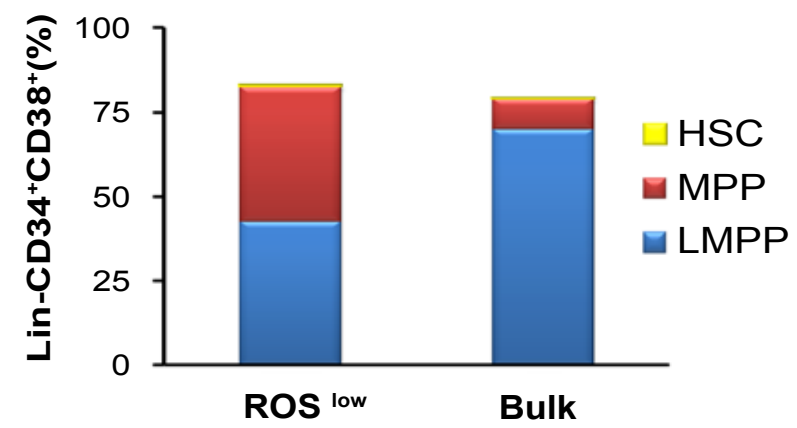

C

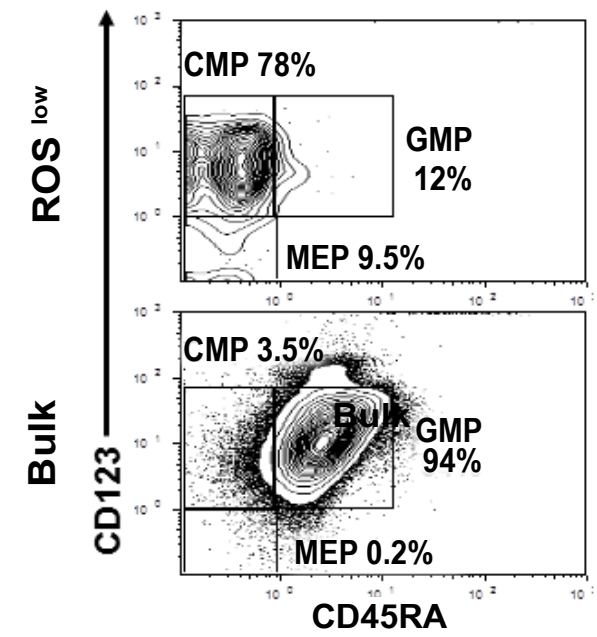

Figure 3: ROS ${ }^{\text {low }}$ cells are significantly enriched in CMP-like cells. (a) Immunophenotypic analysis of the compartment of Lin-CD38+ committed progenitors in 5 patients with LMPP/GMP-like CD34+ AML. The ROS low fraction is highly enriched in CMP-like cells and contains less GMP-like cells compared to the bulk AML population. (b) Analysis of the stem cell compartment of Lin-CD38- cells in the same patients. The ROS low subset contains more MPP-like cells and less LMPP-like cells, but statistical significance is not reached for both comparisons. (c) Representative flow cytometry analysis of leukemic hematopoietic hierarchy in a patient with LMPP/GMP-like CD34+ AML. ROS ${ }^{\text {low }}$ leukemic cells are primarily comprised of CMP-like progenitors and considerably enriched in MEP-like progenitors compared to the bulk CD34+ AML cells. Plots are gated on Lin-CD34+CD38+ cells. P values by paired Student's t-test.

homeostasis is characterized of high complexity [3] thus it appears rather unlikely that a single mutation would be responsible for the observed heterogeneity in ROS levels.

The staining pattern of DCF/DA is typically a continuum of intensities, therefore arbitrary cut-offs are often used to define low, "normal" and high levels of ROS [4,27]. By contrast, we identified a small clonal AML subpopulation with very low redox levels, clearly distinguishable from the bulk AML cells. The prevalence of ROS low cells was not correlated to the total ROS content and, though it was not associated with poor risk features, it was significantly increased in patients with sAML. A possible explanation for this finding is that the oxidative stress in the progenitor cell compartment is more intense in MDS compared to AML and potentially selects for resistant, ROS $^{\text {low }}$ progenitors $[11,29,30]$. As mentioned above, the accurate immunophenotypic characterization of LSCs is controversial. In 
a

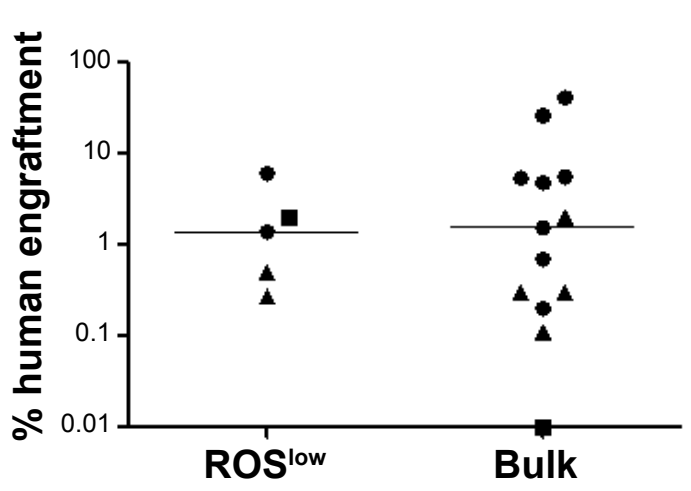

b

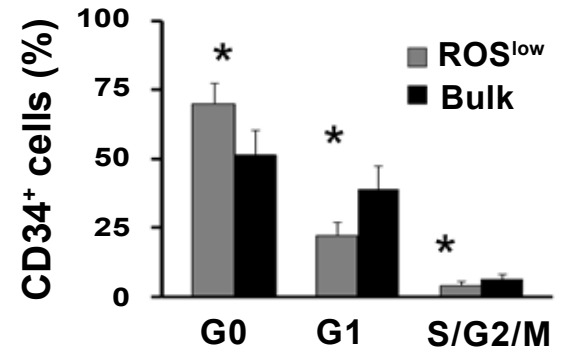

d

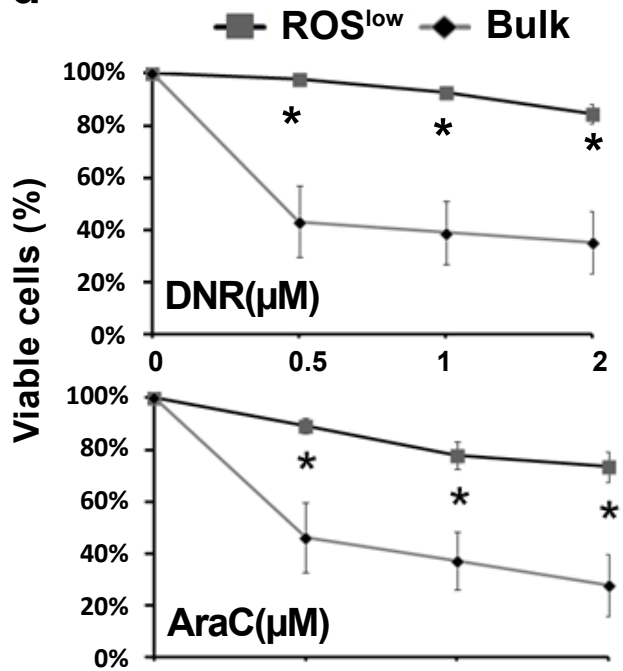

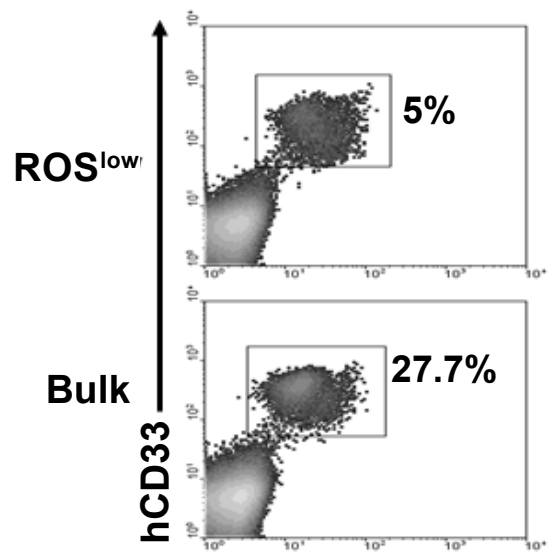

hCD45

C

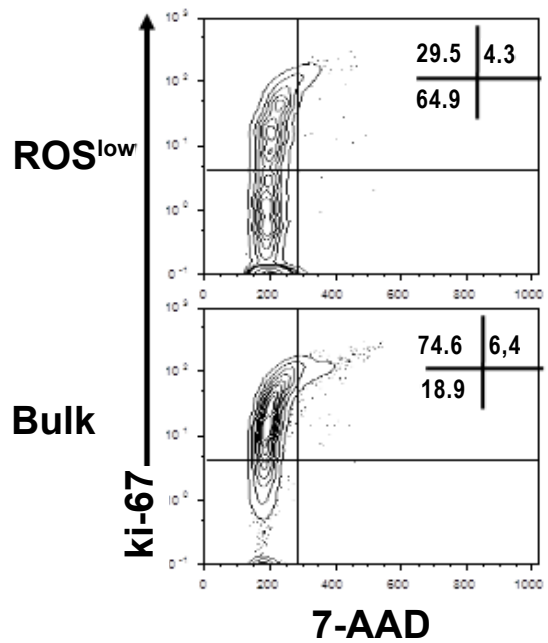

e

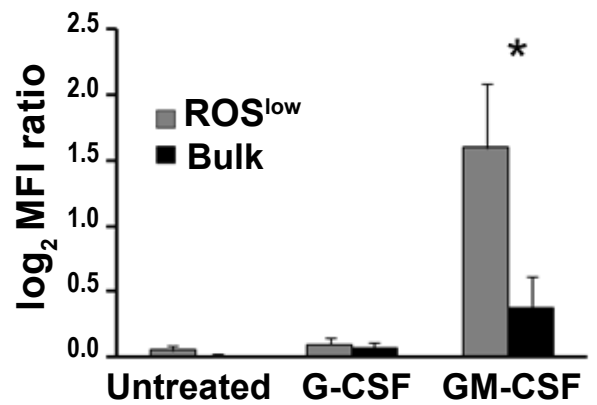

Figure 4: The ROS ${ }^{\text {low }}$ subset bears a chemoresistant phenotype and can establish leukemia in NOD/SCID mice. (a) Primary engraftment of sorted ROS low and total CD34+ AML cells from primary patient samples $(n=3)$ in the bone marrow of NOD/SCID mice (left) Each animal is shown as a dot and animals from the same sample are shown as similar dots. Representative flow cytometry plots of the detection of human CD45/CD33 double positive cells in the bone marrow of a NOD/ SCID mice transplanted with either ROS low or total AML blasts and harvested 12 weeks after transplantation (right). Horizontal bars indicate mean. (b) Cumulative results and representative plots (c) of cell cycle analysis in flow-sorted total and ROS low CD34+ cells ( $\mathrm{n}=5$ ). A significantly higher fraction of ROS ${ }^{\text {low }}$ cells resides in G0 phase, as compared to bulk leukemia cells. By contrast, the latter are more frequently found in G1 and S/G2/M phases. (d) ROS ${ }^{\text {low }}$ cells exhibit increased in vitro chemoresistance in both daunorubicin (DNR, 0.5-2 $\mu \mathrm{M}, \mathrm{n}=4$ ) and cytarabine (AraC, 2.5-10 $\mu \mathrm{M}, \mathrm{n}=4$ ). Viability was normalized to the percentage of viable cells after 24 hours in the control (no drug) cultures and is presented as mean \pm SEM of triplicates. (e) Basal and potentiated phospho-STAT5 expression on sorted ROSlow and total AML blasts $(n=4)$. GM-CSF-induced phosphorylation of STAT5 was significantly higher in ROS ${ }^{\text {low }}$ cells, whereas no differences were observed after G-CSF stimulation. Data are expressed as mean $\pm \mathrm{SEM}$; ${ }^{*} \mathrm{P}<0.05$ by paired Student's t-test

an elegant paper Goardon and colleagues proposed that LSCs in the vast majority of AML patients share progenitor rather than stem cell characteristics, similar to the normal granulocyte-macrophage progenitors (GMP) and the currently poorly characterized lymphoid- primed multipotential progenitor (LMPP) [17]. In 5 patients with LMPP/GMP-like AML we observed that the ROS ${ }^{\text {low }}$ fraction contained less LMPP and GMP-like cells compared to the total leukemic population, whereas it was enriched in MEP-like cells implying a more 
mature phenotype of ROS ${ }^{\text {low }}$ cells. Nevertheless, ROS low cells exhibited comparable primary engraftment capacity in xenotransplantation assays with the bulk leukemia cells. Due to the rarity of ROS ${ }^{\text {low }}$ cells and the rigorous sorting procedure we could not perform secondary transplantations. However, recent data confirm that AML cells with low ROS levels are enriched in LICs [27], although the authors arbitrarily defined ROS $^{\text {low }}$ cells as the $15 \%$ brightest dye fluorescence distribution of DCF, thus encompassing a considerably larger population of AML cells compared to the ROS ${ }^{\text {low }}$ subset in our study [27].

Quiescence and chemoresistance are considered key characteristics of LSCs [31]. Consistent with previous observations, reporting a strong correlation of ROS levels with the cycling status of normal HSCs [11], we found that, in contrast to the total AML cells, almost three-fourths of ROS ${ }^{\text {low }}$ cells were in G0 phase. Additionally, the ROS low subset displayed significantly augmented in vitro chemoresistance to conventional chemotherapeutic agents, which is in line with the reported negative relationship between ROS levels and chemo- and radio resistance of cancer stem cells $[2,8,32]$. The chemoresistant phenotype of ROS ${ }^{\text {low }}$ cells was further corroborated by the over-exuberant response of pSTAT5 after perturbation with GM-CSF, a cell signaling aberration associated with aggressive biologic behavior $[21,22]$.

To conclude, we have identified a novel, biologically distinct ROS ${ }^{\text {low }}$ subpopulation in $\mathrm{CD} 34^{+}$AML. Recent data show that mitochondrial priming via $\mathrm{BH} 3$-only proteins, which engage a ROS-dependent proapoptotic mechanism [33], correlates with the chemosensitivity of AML blasts [34], thus providing a potential explanation for the chemoresistant phenotype of ROS ${ }^{\text {low }}$ cells. Correlational studies of clinical and biological data with the alterations of the ROS ${ }^{\text {low }}$ subset in larger patient cohorts will help to determine the exact role of this novel subpopulation in key management issues in AML, such as prognostic assessment, detection of minimal residual disease and cell-specific therapeutic targeting.

\section{References}

1. Cairns RA, Harris IS, Mak TW (2011) Regulation of cancer cell metabolism. Nat Rev Cancer 11: 85-95. [PubMed]

2. Kobayashi Cl, Suda T (2012) Regulation of reactive oxygen species in stem cells and cancer stem cells. J Cell Physiol 227: 421-430. [PubMed]

3. Zhou F, Shen Q, Claret FX (2013) Novel roles of reactive oxygen species in the pathogenesis of acute myeloid leukemia. J Leukoc Biol 94: 423-439. [PubMed]

4. Jang YY, Sharkis SJ (2007) A low level of reactive oxygen species selects for primitive hematopoietic stem cells that may reside in the low-oxygenic niche. Blood 110: 3056-3063. [PubMed]

5. Yahata T, Takanashi T, Muguruma Y, Ibrahim AA, Matsuzawa H, et al. (2012) Accumulation of oxidative DNA damage restricts the self-renewal capacity of human hematopoietic stem cells. Blood 118: 2941-2950. [PubMed]

6. Ito K, Hirao A, Arai F, Matsuoka S, Takubo K, et al. (2004) Regulation of oxidative stress by ATM is required for self-renewal of haematopoietic stem cells. Nature 431: 997-1002. [PubMed]

7. Tothova Z, Kollipara R, Huntly BJ, Lee BH, Castrillon DH, et al. (2007) FoxOs are critical mediators of hematopoietic stem cell resistance to physiologic oxidative stress. Cell 128: 325-339. [PubMed]

8. Diehn M, Cho RW, Lobo NA, Kalisky T, Dorie MJ, et al. (2009) Association of reactive oxygen species levels and radioresistance in cancer stem cells. Nature 458: 780-783. [PubMed]

9. Rizo A, Olthof S, Han L, Vellenga E, de Haan G, et al. (2009) Repression of BMI1 in normal and leukemic human CD34(+) cells impairs self-renewal and induces apoptosis. Blood 114: 1498-1505. [PubMed]

10. Hole PS, Pearn L, Tonks AJ, James PE, Burnett AK, et al. (2010) Ras-induced reactive oxygen species promote growth factor-independent proliferation in human CD34+ hematopoietic progenitor cells. Blood 115: 1238-1246. [PubMed]
11. Hole PS, Darley RL, Tonks A (2011) Do reactive oxygen species play a role in myeloid leukemias? Blood 117: 5816-5826. [PubMed]

12. Er TK, Tsai SM, Wu SH, Chiang W, Lin HC, et al. (2007) Antioxidant status and superoxide anion radical generation in acute myeloid leukemia. Clin Biochem 40: 1015-1059. [PubMed]

13. Abdel-Wahab O, Levine RL (2010) Metabolism and the leukemic stem cell. J Exp Med 207: 677-680. [PubMed]

14. Zhou FL, Zhang WG, Wei YC, Meng S, Bai GG, et al. (2010) Involvement of oxidative stress in the relapse of acute myeloid leukemia. J Biol Chem 285 15010-15015. [PubMed]

15. Walter RB, Appelbaum FR, Estey EH, Bernstein ID (2012) Acute myeloid leukemia stem cells and CD33-targeted immunotherapy. Blood 119: 61986208. [PubMed]

16. Themeli M, Petrikkos L, Waterhouse M, Bertz H, Lagadinou E, et al. (2010) Alloreactive microenvironment after human hematopoietic cell transplantation induces genomic alterations in epithelium through an ROS-mediated mechanism: in vivo and in vitro study and implications to secondary neoplasia. Leukemia 24: 536-543. [PubMed]

17. Goardon N, Marchi E, Atzberger A, Quek L, Schuh A, et al. (2011) Coexistence of LMPP-like and GMP-like leukemia stem cells in acute myeloid leukemia. Cancer Cell 19: 138-152. [PubMed]

18. Lagadinou ED, Ziros PG, Tsopra OA, Dimas K, Kokkinou D, et al. (2008) C-Jun N-terminal kinase activation failure is a new mechanism of anthracycline resistance in acute myeloid leukemia. Leukemia 22: 1899-1908. [PubMed]

19. Siapati EK, Papadaki M, Kozaou Z, Rouka E, Michali E, et al. (2011) Proliferation and bone marrow engraftment of AML blasts is dependent on beta-catenin signalling. Br J Haematol 152: 164-174. [PubMed]

20. Wartenberg M, Fischer K, Hescheler J, Sauer H (2000) Redox regulation of P-glycoprotein-mediated multidrug resistance in multicellular prostate tumor spheroids. Int J Cancer 85: 267-274. [PubMed]

21. Irish JM, Hovland R, Krutzik PO, Perez OD, Bruserud O, et al. (2004) Single cell profiling of potentiated phospho-protein networks in cancer cells. Cell 118 217-228. [PubMed]

22. Heuser M, Sly LM, Argiropoulos B, Kuchenbauer F, Lai C, et al. (2009) Modeling the functional heterogeneity of leukemia stem cells: role of STAT5 in leukemia stem cell self-renewal. Blood 114: 3983-3993. [PubMed]

23. Kotecha N, Flores NJ, Irish JM, Simonds EF, Sakai DS, et al. (2008) Singlecell profiling identifies aberrant STAT5 activation in myeloid malignancies with specific clinical and biologic correlates. Cancer Cell 14: 335-343. [PubMed]

24. Horton SJ, Huntly BJ (2012) Recent advances in acute myeloid leukemia stem cell biology. Haematologica 97: 966-974. [PubMed]

25. Lutz C, Hoang VT, Buss E, Ho AD (2013) Identifying leukemia stem cells--is it feasible and does it matter? Cancer letters 338: 10-14. [PubMed]

26. Bonnet D, Dick JE (1997) Human acute myeloid leukemia is organized as a hierarchy that originates from a primitive hematopoietic cell. Nat Med 3: 730737. [PubMed]

27. Lagadinou ED, Sach A, Callahan K, Rossi RM, Neering SJ, et al. (2013) BCL2 Inhibition Targets Oxidative Phosphorylation and Selectively Eradicates Quiescent Human Leukemia Stem Cells. Cell stem cell 12: 329-341. [PubMed]

28. Sallmyr A, Fan J, Datta K, Kim KT, Grosu D, et al. (2008) Internal tandem duplication of FLT3 (FLT3/ITD) induces increased ROS production, DNA damage, and misrepair: implications for poor prognosis in AML. Blood 111: 3173-3182. [PubMed]

29. Bowen D, Wang L, Frew M, Kerr R, Groves M (2003) Antioxidant enzyme expression in myelodysplastic and acute myeloid leukemia bone marrow: further evidence of a pathogenetic role for oxidative stress? Haematologica 88: 1070-1072. [PubMed]

30. Chung YJ, Robert C, Gough SM, Rassool FV, Aplan PD (2014) Oxidative stress leads to increased mutation frequency in a murine model of myelodysplastic syndrome. Leuk Res 38: 95-102. [PubMed]

31. Buss EC, Ho AD (2011) Leukemia stem cells. Int J Cancer 129: 2328-2336. [PubMed]

32. Wang J, Yi J (2008) Cancer cell killing via ROS: to increase or decrease, that is the question. Cancer Biol Ther 7: 1875-1884. [PubMed] 
Citation: Kotsianidis I, Kokkinou D, Siapati EK, Miltiades P, Lamprianidou E, et al. (2014) Identification of a Chemoresistant "Oxidative State-Low" Leukemic Subpopulation in CD34+ Human Acute Myeloid Leukemia. J Stem Cell Res Ther 4: 235. doi:10.4172/2157-7633.1000235

Page 9 of 9

33. Garcia-Perez C, Roy SS, Naghdi S, Lin X, Davies E, et al. (2012) Bid-induced mitochondrial membrane permeabilization waves propagated by local reactive oxygen species (ROS) signaling. Proc Natl Acad Sci USA 109: 4497-4502. [PubMed]
34. Vo TT, Ryan J, Carrasco R, Neuberg D, Rossi DJ, et al. (2012) Relative Mitochondrial Priming of Myeloblasts and Normal HSCs Determines Chemotherapeutic Success in AML. Cell 151: 344-355. [PubMed] 